\title{
Experiments on the interaction between long Josephson junctions and a coplanar strip resonator
}

Davidson, A.; Pedersen, Niels Falsig

Published in:

Applied Physics Letters

Link to article, DOI:

$10.1063 / 1.107129$

Publication date:

1992

Document Version

Publisher's PDF, also known as Version of record

Link back to DTU Orbit

Citation (APA):

Davidson, A., \& Pedersen, N. F. (1992). Experiments on the interaction between long Josephson junctions and a coplanar strip resonator. Applied Physics Letters, 60(16), 2017-2019. https://doi.org/10.1063/1.107129

\section{General rights}

Copyright and moral rights for the publications made accessible in the public portal are retained by the authors and/or other copyright owners and it is a condition of accessing publications that users recognise and abide by the legal requirements associated with these rights.

- Users may download and print one copy of any publication from the public portal for the purpose of private study or research.

- You may not further distribute the material or use it for any profit-making activity or commercial gain

- You may freely distribute the URL identifying the publication in the public portal 


\title{
Experiments on the interaction between long Josephson junctions and a coplanar strip resonator
}

\author{
A. Davidson \\ IBM Research Division, T. J. Watson Research Center, Yorktown Heights, New York 10598
}

N. F. Pedersen

Physics Laboratory I, Technical University of Denmark, DK-2800 Lyngby, Denmark

(Received 5 September 1991; accepted for publication 4 February 1992)

\begin{abstract}
Experiments are reported on a new geometry designed to couple long Josephson junction fluxon oscillators to a resonant cavity. The junctions were made with a niobium-aluminum oxideniobium trilayer process with a critical-current density of around $1000 \mathrm{~A} / \mathrm{cm}^{2}$. Various numbers of such junctions were incorporated directly as part of a coplanar strip half-wave resonator, with fundamental mode of about $34 \mathrm{GHz}$. Both the current density and oscillation frequency are higher than in previous experiments. Evidence for phase locking of multiple junctions is presented.
\end{abstract}

The study of phase locking of fluxon oscillations in long Josephson tunnel junctions has been stimulated by potential electronic applications of these devices. ${ }^{1}$ Seriesbiased arrays of long junctions operating in the resonant fluxon oscillation mode are serious candidates for employment as local oscillators in integrated superconducting microwave and millimeter wave circuits. Operating such an array in a coherent, phase-locked mode offers increased output power and reduced oscillator linewidth with respect to the numbers obtainable from a single junction oscillator. A resonator or cavity is very useful to enhance the coupling between several long junctions as was shown by experiment ${ }^{2}$ and numerical ${ }^{3,4}$ work. In this letter we report experiments on the interaction between several long Josephson junctions and a different type of superconducting cavity: a coplanar strip resonator. A preliminary part of this investigation was presented earlier. ${ }^{5}$

A series of high-quality samples were fabricated in a $\mathrm{Nb} / \mathrm{AlO}_{x} / \mathrm{Nb}$ trilayer technology with a Josephson critical-current density of about $1000 \mathrm{~A} / \mathrm{cm}^{2}$, the highest yet for soliton oscillator experiments. The process is described elsewhere. ${ }^{6,7}$ As described in Ref. 5, two different lengths of junctions were used, $200 \mu \mathrm{m}$ (corresponding to a normalized length $\ell$ of about 20), and $80 \mu \mathrm{m}(\ell \propto 8)$. All junctions were $10 \mu \mathrm{m}$ wide-roughly the Josephson penetration depth. The junctions and coplanar ${ }^{8}$ structure are shown in Fig. 1. There are two parallel strips of niobium, each $2000 \mu \mathrm{m}$ long, $200 \mu \mathrm{m}$ wide, and $1500 \AA$ thick, deposited on an oxidized silicon wafer. These strips were parallel along their long axes, and were separated by 44 $\mu \mathrm{m}$. The design of the resonator was for a fundamental half-wave frequency of $30 \mathrm{GHz}$ and a characteristic impedance of $60 \Omega$.

This fundamental resonant frequency was confirmed ${ }^{9}$ by direct measurements. Radiation from a room-temperature source was introduced into the cryostat, and the suppression of the junctions' supercurrent was measured as a function of frequency. The fundamental mode was measured to be $34.45 \mathrm{GHz}$, with a $Q$ of about 350 at $4.2 \mathrm{~K}$. This is consistent with the design frequency, since small changes may occur due to geometrical details not taken into account in the calculations. We also point out that the geometry allows for other resonant structures such as a microstrip resonator between one of the electrodes and a ground plane below the substrate.

The following types of sample geometries were fabricated and tested, with both 200 and $80 \mu \mathrm{m}$ junctions: (A) samples with $2,4,8$, and 16 long junctions closely spaced near the center of one of the electrodes of the coplanar waveguide (sce Fig. 1); (B) samples with 16 junctions uniformly distributed on one of the electrodes of the coplanar waveguide; and (C) samples with 32 junctions uniformly distributed on both of the electrodes of the coplanar waveguide.

The simplest case is a two junction sample of type A. Figure 2 shows some $I-V$ curves obtained for such a sample with junctions of length $200 \mu \mathrm{m}$. The bias current passes through both junctions, and only the sum of the voltages can be measured (see Fig. 1). Normally the voltage $V_{n}$ for an $n$ fluxon state is approximately proportional to $n$. In Fig. 2 we have chosen to normalize the measured voltage by the total number of fluxons in the two junctions. This is done in order to facilitate comparison between the different fluxon states. Hence $V_{n} / n$ is plotted horizontally in Fig. 2. For these samples with longer junctions the asymptotic voltage for the one fluxon curve is about $100 \mu \mathrm{V}$. From this we infer a specific capacitance for our junctions of $0.042 \mathrm{pF} / \mu \mathrm{m}^{2}$, and a phase velocity of $9.6 \mu \mathrm{m} / \mathrm{ps}$, about $3 \%$ of the speed of light.

Figure 2(a) shows the $I-V$ curve with one junction in a one fluxon state and the other in the zero voltage state;

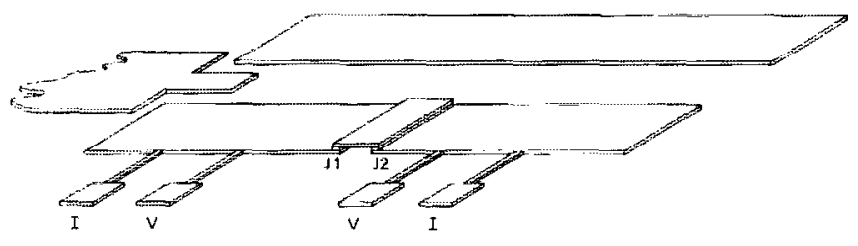

FIG. 1. Schematic drawing of the sample geometry. The junctions $\mathrm{J} 1$ and $\mathrm{J} 2$ are shown above the coplanar strip for clarity. In the actual samples, they were below. 


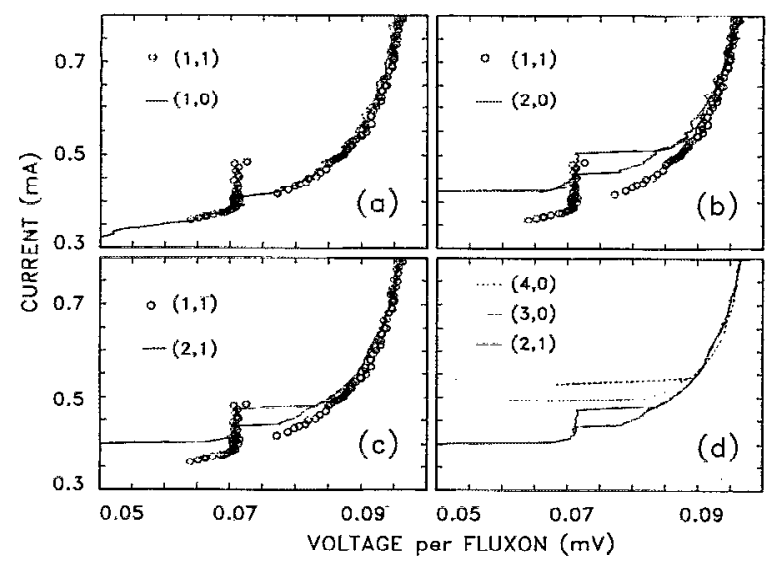

FIG. 2. Measured $I$ - $V$ curves for the different types of excitations in the two junction samples (type A). The voltages are shown normalized by the number of fluxons (voltage per fluxon) to facilitate comparison.

we refer to this as the $(1,0)$ mode. A small step is observed at $72 \mu \mathrm{V}$ corresponding to a fluxon oscillation frequency of $17.4 \mathrm{GHz}$, the first subharmonic of the cavity resonance at $34.8 \mathrm{GHz}$. (This value of the fundamental resonator mode differs by less than one percent from that obtained by the direct microwave method, and is wcll within the calibration errors of our amplifier.) Note again, that in Fig. 2, all curves are displayed as the voltage per fluxon $V_{n} / n$. For example, the line in Fig. 2(c) for the $(2,1)$ state represents the measured voltage divided by three.

Figure 2(a) also shows a $(1,1)$ mode corresponding to one fluxon in each of the two junctions. This state was obtained by increasing the bias current above the critical current until both junctions switched to the energy gap voltage $2 \Delta / e$. Then the current was slowly and carefully decreased (through many higher order fluxon modes) until the voltage was about twice that of the $(1,0)$ mode. Because of the very high sample uniformity, this procedure always gave a state with one fluxon in each of the junctions. The voltage for the $(1,1)$ mode is exactly twice that of the $(1,0)$ mode, except for the much larger step at 72 $\mu V$. The two fluxons in the two junctions add their contributions to the voltage and do not interact with each other except for frequencies where the cavity mode is a mediator. This interaction of the solitons in different junctions through the cavity is reflected in the size of the $(1,1)$ step, which is more than twice that of the $(1,0)$ step. (Exactly a factor of two was expected. $)^{2-4}$

Figure $2(\mathrm{~b})$ shows the $(2,0)$ mode [with the $(1,1)$ mode again for reference] corresponding to two fiuxons in one junction and none in the other. This state is obtained by increasing the bias current slowly so that one junction is switched to the voltage $2 \Delta / e$ while the other remains in the zero voltage state. By now decreasing the current, the junction steps through the $(n, 0)$ modes, so the $(2,0)$ mode may be selected. Two different fluxon configurations with the following signatures are possible: (i) a bunched ${ }^{10}$ two soliton mode $(2 \mathrm{~B}, 0)$ with roughly the same oscillator frequency as the $(1,0)$ mode, the same coupling to the 34.8 $\mathrm{GHz}$ resonance frequency, and the same locking range and
$I$ - $V$ curve; or (ii) a symmetric mode $(2 \mathrm{~S}, 0)$ with a fluxon and an antifluxon in one junction moving in opposite directions, with twice the frequency of the $(1,0)$ modes, coupled more directly to the cavity fundamental, but with reduced $Q$, and with a reduced voltage and lock range due to extra soliton-antisoliton collisions. Thus the locking range is expected to be somewhat smaller than that for the $(2 \mathrm{~B}, 0)$ mode. The experimental $I-V$ curve of Fig. $2(\mathrm{~b})$ has the signature of (ii), corresponding to the symmetric motion of the $(2 \mathrm{~S}, 0)$ mode. That this type of motion typically takes place at the bottom of an $n=2$ step is known from numerical simulation ${ }^{10}$ and microwave experiments. ${ }^{11}$

Figure 2(c) shows the $(2,1)$ mode with the $(1,1)$ mode as a reference, obtained just as described above. For the $(2,1)$ mode, the background voltage is just the sum of the voltages for the $(2,0)$ mode and the $(1,0)$ mode, as can be expected. Since the two junctions interact with the cavity only at harmonic frequencies, the locking range is similar to the largest of the individual locking ranges, that of the $(2 \mathrm{~S}, 0)$ mode as can be seen by comparing with Fig. 2(b).

For the $(3,0)$ mode, Fig. 2(d), the collisions have further reduced the average voltage, and upon decreasing the bias current the fluxons annihilate before the bias range for phase locking has been reached. The mode observed is most likely of the $(3 \mathrm{~S}, 0)$ type rather than of the $(3 \mathrm{~B}, 0)$ type. The same features apply to the $(4,0)$ mode also shown in Fig. 2(d).

Experiments with four junctions $(200 \mu \mathrm{m})$ closely spaced near the center of one of the electrodes of the coplanar waveguide showed the following features: (1) the critical currents of the four junctions were very different. This was not because of junction defects or fabrication nonuniformity, but most likely because of self-field effects connected with geometry. This effect was never observed with the two junction samples; and (2) because of the above-mentioned difference in critical currents, the locking range never exceeded that of the two junction samples. The same features applied to the closely spaced eight junction samples.

Another series of experiments was done on the 16 junction samples with the junction pairs not so close to each other, but evenly distributed over the length of one of the electrodes of coplanar the cavity (samples of type B). In this case it was possible to have rather uniform critical currents in all the junctions. Probably the self-fields were eliminated because of the larger distance between the junctions.

Figure 3 is an experimental $I-V$ curve for a type B sample with $200 \mu \mathrm{m}$ junctions. It shows the locking of up to 14 fluxons in 14 different junctions. The 15 th and higher steps are markedly different (as can be seen at the bottom of steps) and show a decreasing locking range. This is probably because multiple fluxons appear in some of the junctions, which leads to interactions of the nature discussed above, and a reduced locking range. The largest locking range is about $100 \mu \mathrm{A}$, comparable to the locking ranges in Fig. 2. Note that the high uniformity of the junctions is reflected in the small spread of the return points al 


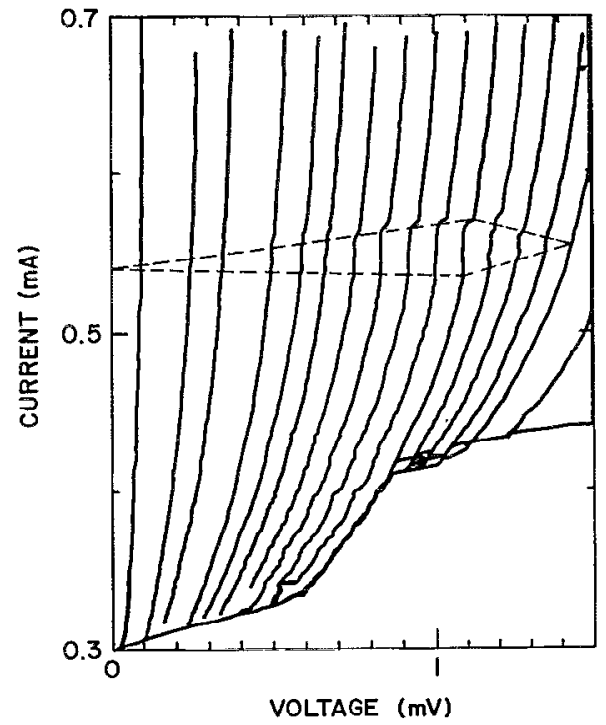

FIG. 3. In $V$ curves for a 16 junction sample (type B). The dashed lines indicate the locking range. Note that the current scale begins at $0.3 \mathrm{~mA}$, and not at 0 .

the bottom of the steps. From Fig. 3 we note that the locking range increases linearly with the number of locked junctions. This phenomenon was first observed in Ref. 2, where it was also confirmed by numerical simulation. By a simple argument this is to be expected. If all the single solitons in each of the junctions are locked, the voltage input to the cavity is proportional to the number of junctions. As a general observation the locking range (for external radiation) seems to go as the square root of the applied power, ${ }^{12}$ in agreement with Fig. 3.

The 16 junctions used in Fig. 3 were all on one side of the coplanar cavity as shown in Fig. 1. In another sample 32 junctions were evenly distributed on both electrodes of the coplanar waveguide (type $C$ ). To our surprise no phase locking at all was observed. The direction of the bias currents on the two sides could be changed, but this had no effect at all.

For completeness it is mentioned that in one of the samples, the junctions were coupled much better to the cavity than what is shown above. A cavity induced step of more than $1 \mathrm{~mA}$ was observed (for a critical current of about $10 \mathrm{~mA}$ ). The reason for this is not yet understood. The shorter samples with a length of $80 \mu \mathrm{m}$ were also investigated in all the configurations shown for the $200 \mu \mathrm{m}$ samples. In many cases phase locking to the cavity was observed, but the fluxon steps were generally fewer and flatter, resembling those of electromagnetic waves in the junctions themselves. This is consistent with their shorter lengths of about eight penetration depths.

In summary, a series of samples designed to investigate the locking of several long junctions to a coplanar strip resonator has been designed and tested. Details of the interactions were possible to resolve on some cases. For weak coupling to the cavity, locking of as many as 14 junctions to a cavity mode (and therefore to each other) was observed. This work has shown phase-lock effects with higher current-density junctions oscillating at a higher frequency than earlier experiments, and is encouraging for millimeter wave applications.

This work was partially conducted under the auspices of the Consortium for Superconducting Electronics (CSE). The niobium trilayers were provided by M.I.T. Lincoln Laboratory with support under the CSE from the Defense Advanced Research Projects Agency (Contract No. MDA972-90-C-0021). We thank the authors of Refs. 6 and 7 and the IBM Yorktown Silicon Facility for fabrication of the chips.

'N. F. Pedersen, IEEE Trans. Magn. MAG-27, 3328 (1991).

${ }^{2}$ R. Monaco, N. Gronbech-Jensen, and R. D. Parmentier, Phys. Lett. 151, 195 (1991).

${ }^{3}$ G. Filatrella, N. Gronbech-Jensen, R. Monaco, S. Pagano, R. D. Parmentier, N. F. Pedersen, G. Rotoli, M. Salerno, and M. R. Samuelsen, Procecdings of the NATO Advanced Reseurch Workshop on Superconducting Electronics, Capri 1990 (Plenum, London, 1991) (to be published).

${ }^{4}$ N. Gronbech-Jensen, N. F. Pedersen, A. Davidson, and R. D. Pamentier, Phys. Rev. B 42, 6035 (1990).

${ }^{5}$ A. Davidson, N. Gronbech-Jensen, and N. F. Pedersen, IEEE Trans. Magn. MAG-27, 3347 (1991).

${ }^{6}$ M. B. Ketchen et al., Appl. Phys. Lett. 59, 2609 (1991).

${ }^{7}$ M. Bhushan and E. M. Macedo, Appl. Phys. Lett. 28, 1323 (1991).

${ }^{8}$ C. P. Wen, IEEE Trans. Microwave Theory Tech. MTT-17, 1087 (1969).

${ }^{4}$ P. Barbara, A. Davidson, J. Holm, J. Mygind, and N. F. Pedersen (unpublished).

${ }^{10}$ S. N. Erne and R. D. Parmentier, IEEE Trans. Mag. MAG-17, 804 (1981).

${ }^{11}$ B. Dueholm, O. A. Levring, J. Mygind, N. F. Pedersen, O. H. Soerensen, and M. Cirillo, Phys. Rev. Lett. 46, 1299 (1981).

${ }^{12}$ M. Cirillo and F. L. Lloyd, J. Appl. Phys. 61, 2581 (1987). 